\title{
Novel Chest Wall Reconstruction Following Excision of an Xiphisternal Chondrosarcoma
}

\author{
Miranda C. Hann, MD, ${ }^{1}$ Brian Pettiford, MD, ${ }^{2}$ Christopher Babycos, MD ${ }^{1,3}$ \\ ${ }^{1}$ The University of Queensland School of Medicine, Ochsner Clinical School, New Orleans, LA ${ }^{2}$ Department of Thoracic Surgery, \\ Ochsner Clinic Foundation, New Orleans, LA ${ }^{3}$ Department of Plastic Surgery, Ochsner Clinic Foundation, New Orleans, LA
}

\begin{abstract}
Background: Chondrosarcoma, the most common primary malignant tumor of the chest wall, most frequently arises from the sternum, with limited reported cases of tumor origination from the xiphoid process. Because of the location, patients present with complaints of a large chest wall mass associated with pain and respiratory symptoms. These tumors are best managed by en bloc resection and chest wall reconstruction.

Case Report: A 49-year-old male nonsmoker with a strong family history of colon cancer presented with a large, painful chest wall mass associated with shortness of breath and exertional chest pressure. Computed tomography scan demonstrated a large mass arising from the xiphoid process; biopsy confirmed a grade 1 chondrosarcoma. The mass was removed en bloc and repaired with an innovative technique using omentum and PROCEED Surgical Mesh (Ethicon US, LLC). The wound was closed with a fasciocutaneous flap.

Conclusion: To our knowledge, this case is the first report of a chest wall reconstruction of a large inferior sternal defect using only polypropylene and omentum without a rigid support or muscular flap. This repair option could be considered for patients in whom traditional rigid repair methods have potential complications or limitations.
\end{abstract}

Keywords: Chondrosarcoma, omentum, polypropylene, surgical flaps

Address correspondence to Brian Pettiford, MD, Department of Thoracic Surgery, Ochsner Clinic Foundation, 1514 Jefferson Hwy., New Orleans, LA 70121. Tel: (504) 842-3966. Email: brian.pettiford@ochsner.org

\section{INTRODUCTION}

Chondrosarcoma, a malignant tumor arising from cartilage, is the most common primary malignancy of the chest wall and represents $20 \%$ of all chest wall neoplasms. ${ }^{1}$ These tumors are rarely found arising from the xiphoid, with only 4 previously reported cases. ${ }^{2}$ In these cases, the tumors grew caudally into the soft tissue below the xiphoid. Patients often present with a painful chest wall mass and respiratory symptoms from compression. Early recognition with a wide en bloc oncologic resection of the tumor results in the best prognosis. We report the case of a patient with a chondrosarcoma that was removed en bloc, followed by a modified chest wall reconstruction.

\section{CASE REPORT}

A 49-year-old male nonsmoker with a strong family history of colon cancer and a 1-year history of an enlarging central chest mass presented to the emergency department (ED) with chest pain, chest tightness, and shortness of breath. This ED visit was the patient's first presentation, and imaging showed a $7-\mathrm{cm}$, firm, immobile, nontender, nonerythematous mass extending from the lower sternum and xiphoid into the epigastrium.

Computed tomography (CT) scan showed a large tumor adjacent to the pericardium, compressing the right ventricle and extending into the soft tissues anteriorly (Figure 1). Core needle biopsy of the mass revealed a grade 1 chondrosarcoma, and positron emission tomography scan showed mild hypermetabolism within the mass with no evidence of metastatic disease.

At the multidisciplinary oncologic lung conference, the decision was made to proceed with en bloc resection. Resection involved a partial sternectomy, including an en bloc resection of the diaphragm, pericardium, and costal cartilage surrounding the mass (Figure 2). Pathology confirmed that negative margins were obtained. The superior epigastric vessels and internal mammary vessels were ligated bilaterally because of tumor size, precluding rectus reconstruction. Both phrenic nerves were preserved.

The final defect measured $25 \mathrm{~cm}$ laterally and $15 \mathrm{~cm}$ craniocaudally. The plastic surgery team performed an omental flap and mesh reconstruction of the chest defect (Figure 3). The omentum was freed from the peritoneal cavity as a vascularized pedicle and used to fill the defect and cover the anterior surface of the heart. The omentum was secured to surrounding tissue with 2-0 absorbable suture. PROCEED Surgical Mesh (Ethicon US, LLC), a polypropylene and laminate mesh soaked in antibiotic solution, was used as a tight covering over the omentum to reconstruct the chest wall. It 


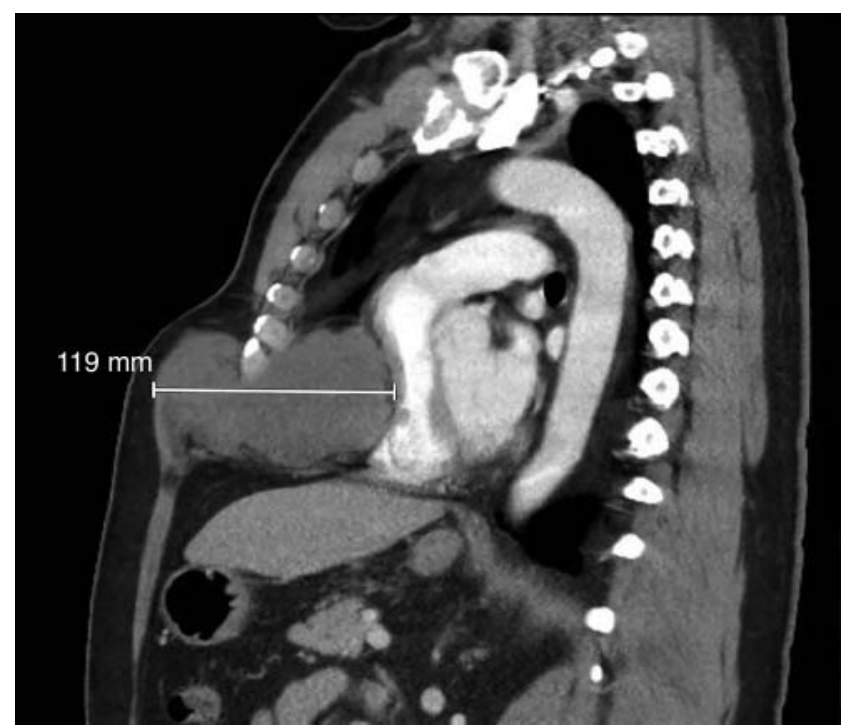

Figure 1. Sagittal computed tomography of the chest shows the chondrosarcoma anteriorly. A fat plane was visible between the mass and pericardium. The mass is compressing the right ventricle and extending into the soft tissues anteriorly.

was secured superiorly to the pectoralis major muscles and inferiorly to the rectus muscles. The diaphragm was closed tightly around the omentum using polydioxanone sutures to prevent herniation of abdominal contents. The large skin defect was closed after elevation and rotation of a fasciocutaneous flap. Bilateral chest tubes were placed to drain serous fluid postoperatively.

The mass measured $14.5 \mathrm{~cm}$, resection margins were clear, and all sampled lymph nodes were benign. The patient had stage T2NOMX ( $>8 \mathrm{~cm}$ in greatest dimension with no lymph node involvement or distant metastases) chondrosarcoma, grade 1.

Postoperatively, the patient was extubated to bilevel positive airway pressure to support adequate lung expansion. The chest tubes were removed uneventfully on postoperative day 3 . After the patient's discharge, he required 2 thoracocenteses to drain pleural effusions: on postoperative day $15,1.6 \mathrm{~L}$ of serosanguinous fluid was drained and on postoperative day 22, $900 \mathrm{cc}$ of serosanguinous fluid was drained. The patient recovered heart and lung function postoperatively and has been counseled about the risks of blunt or sharp anterior chest trauma.

\section{DISCUSSION}

Chondrosarcomas represent $0.5 \%-1 \%$ of all neoplasms and $20 \%$ of chest wall neoplasms, the most common primary malignant tumor of the chest wall. ${ }^{1}$ Researchers at the Memorial Sloan-Kettering Cancer Center reviewed 40 years of patient history and reported primary location occurrence incidence rates of $5 \%$ in the clavicles, $16 \%$ in the sternum, $36 \%$ in the scapula, and $43 \%$ in the ribs. ${ }^{1}$ In reported xiphoid cases, the tumors grew inferiorly. Because our patient presented for the first time to the ED with a full-sized tumor, evolution of his chondrosarcoma can be hypothesized as radial based on location, size, and erosion into the pericardium; however, this hypothesis cannot be confirmed as the evolution of the tumor is unknown.

The treatment of choice for thoracic wall chondrosarcoma is radical en bloc excision with wide margins because chondrosarcomas are usually resistant to chemotherapy. ${ }^{3}$ One of the major challenges after tumor resection is reconstruction of the chest wall. The goal is to create an adequate or rigid thoracic cage for chest wall stability, good pulmonary function, and protection of thoracic organs. Various materials have been used in an attempt to achieve adequate stability. Often a rigid repair material, such as methyl methacrylate cement, muscular flaps, titanium rib and sternal plates, or cryopreserved bone graft, are used to mimic the original chest wall stability. ${ }^{4-6}$ The drawbacks of methyl methacrylate include wound complications, tilting, excursion of the plate if it is not properly fixed, and failure to create an adequate scaffold for new bone. ${ }^{3}$ In our case, we had concerns about the exothermic properties of the methyl methacrylate and migration of the plate through the omentum and into the exposed right ventricle; thus, we chose to avoid this method of repair. Cryopreserved bone grafts are easily manipulated and tailored to a site but can be hard to access and are time consuming to fixate. ${ }^{4}$ Our hospital did not

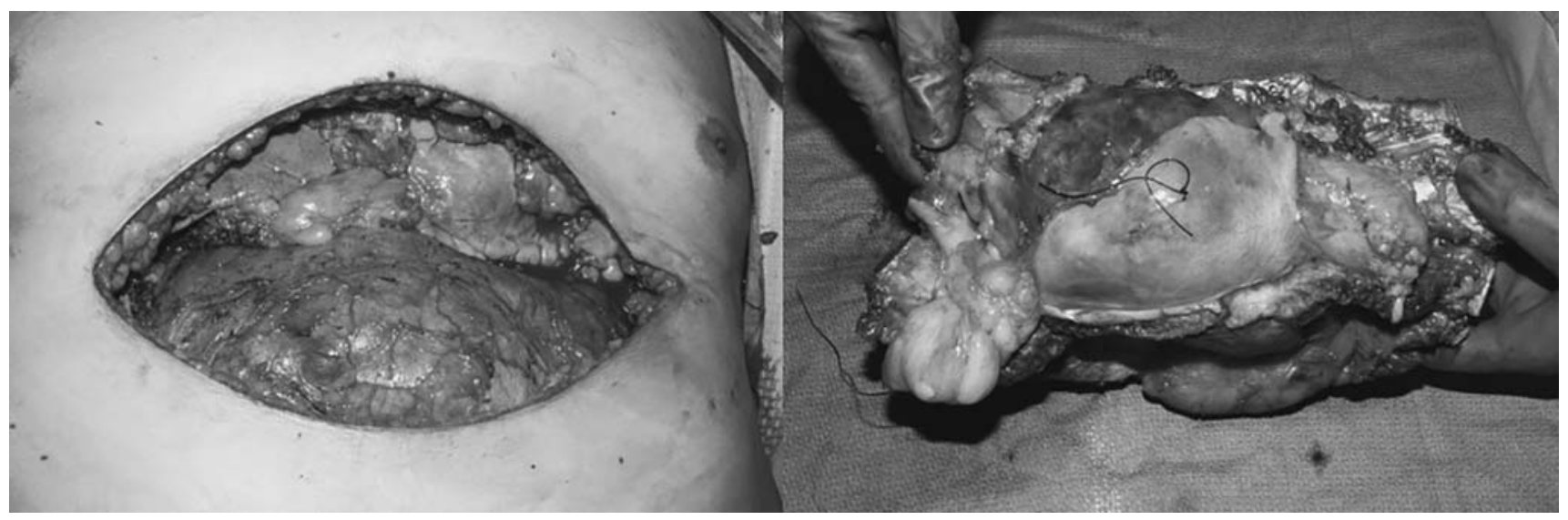

Figure 2. The chest defect (left) and the resected chondrosarcoma (right). Ribs 7 and 8 costal cartilages were dissected and transected. Peristernal dissection was carried out superior to the tumor and was transected with a sternal saw. The tumor was adherent to the anterior pericardium, which was resected from the surface of the heart. 


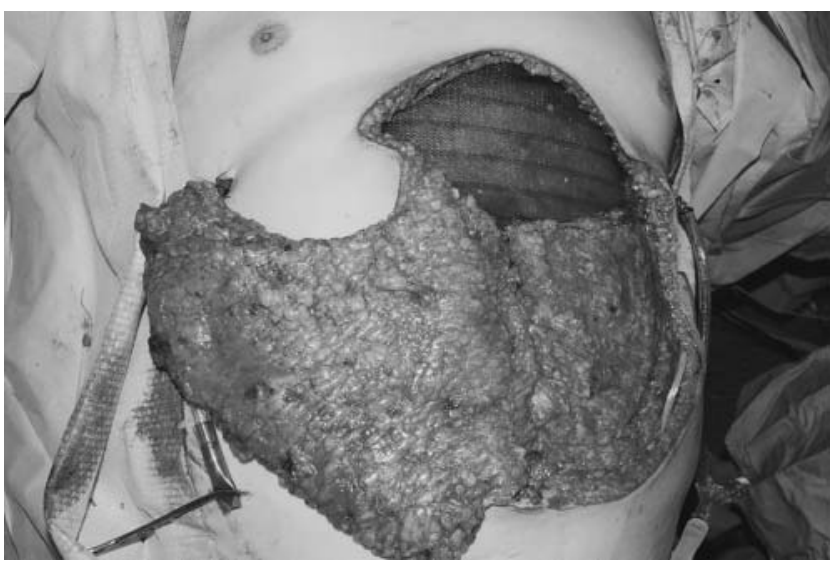

Figure 3. The omentum was freed from the peritoneal cavity and used to fill the defect and cover the heart. PROCEED Surgical Mesh (Ethicon US, LLC), a polypropylene and laminate mesh, was used to reconstruct the chest wall and provide semirigid coverage. The large skin defect was closed after elevation and rotation of a fasciocutaneous flap.

have access to cryopreserved bone graft at the time of the operation. Titanium has been used to help create rigid support across anterior chest wall dissections and can be imaged with CT scan and magnetic resonance imaging, allowing safe postoperative surveillance. ${ }^{7}$ However, the available titanium plates were not long enough to span our patient's defect.

Combined with rigid support, other materials are often used as barriers or added support layers, such as omentum, polypropylene mesh, and polytetrafluoroethylene patches. The omentum is vascularized, which can help carry antibiotics to the defect site, and angiogenic, amorphous in shape, and bulky. ${ }^{8}$ The omentum is often used to cover rigid chest wall replacements, such as methyl methacrylate cement, to prevent infection and create a bed for skin grafting. ${ }^{9}$ Omental flaps are a particularly excellent option for filling large inferior sternal defects. ${ }^{10} \mathrm{~A}$ pectoralis turnover flap could also provide coverage to this area, but this approach was not an option for our patient because of ligation of the internal mammary arteries during dissection. Complications such as herniation, spread of abdominal infection, and omental pedicle necrosis are rare. ${ }^{9}$ Polypropylene is often used to repair hernia-like defects but can be a nidus for infection and may wrinkle during placement. ${ }^{11}$ In our patient, we used omentum as an allogenic covering for the heart and exposed lungs, creating a bulky cushioned barrier to fill the defect, and covering it with a tightly placed polypropylene mesh that created a semirigid barrier for containing the thoracic organs.

To our knowledge, our case is the first report of a chest wall reconstruction of a large defect using a combination of polypropylene and omentum without a rigid support or muscular flap. This repair option could be used as a treatment option for patients in whom rigid chest wall reconstruction for large defects is not possible or available.

\section{CONCLUSION}

Our patient presented with a unique chondrosarcoma arising from the xiphoid and eroding into the pericardium. Performing a reconstruction using only omentum and polypropylene could be a novel and effective means of anterior inferior chest wall reconstruction of large defects, particularly in situations when traditional rigid repair methods have potential complications.

\section{ACKNOWLEDGMENTS}

The authors have no financial or proprietary interest in the subject matter of this article.

\section{REFERENCES}

1. Burt $M$, Fulton $M$, Wessner-Dunlap $S$, et al. Primary bony and cartilaginous sarcomas of chest wall: results of therapy. Ann Thorac Surg. 1992 Aug;54(2):226-232.

2. Aoki J, Moser RP Jr, Kransdorf MJ. Chondrosarcoma of the sternum: CT features. J Comput Assist Tomogr. 1989 Sep-Oct;13 (5):806-810. doi: 10.1097/00004728-198909000-00010.

3. Chaudhry lu, Alhajji Z, Aldulaijan F, Mutairi H, Amr SS. Manubrioclavicular and manubriosternal reconstruction after radical resection for chondrosarcoma of manubriosternum: a modified surgical technique. Ann Thorac Surg. 2015 Jun;99(6): e137-e139. doi: 10.1016/j.athoracsur.2015.02.119.

4. Nosotti M, Rosso L, Mendogni $P$, et al. Sternal reconstruction for unusual chondrosarcoma: innovative technique. J Cardiothorac Surg. 2012 May 2;7:40. doi: 10.1186/1749-8090-7-40.

5. Arnold PG, Pairolero PC. Chest-wall reconstruction: an account of 500 consecutive patients. Plast Reconstr Surg. 1996 Oct;98 (5):804-810. doi: 10.1097/00006534-199610000-00008.

6. Chapelier A. Resection and reconstruction for primary sternal tumors. Thorac Surg Clin. 2010 Nov;20(4):529-534. doi: 10.1016/j.thorsurg.2010.06.002.

7. Berthet JP, Canaud L, D'Annoville T, Alric P, Marty-Ane CH. Titanium plates and Dualmesh: a modern combination for reconstructing very large chest wall defects. Ann Thorac Surg. 2011 Jun;91(6):1709-1716. doi: 10.1016/j. athoracsur.2011.02.014.

8. Cusimano LA, Wolfe ET, Tan P, Babycos CR, Bansal A. Left ventricular assist device salvage with omental flap. Plast Reconstr Surg Glob Open. 2017 Mar 1;5(3):e1250. doi: 10.1097/ GOX.0000000000001250.

9. Shrager JB, Wain JC, Wright CD, et al. Omentum is highly effective in the management of complex cardiothoracic surgical problems. J Thorac Cardiovasc Surg. 2003 Mar;125 (3):526-532. doi: 10.1067/mtc.2003.12.

10. Izaddoost $\mathrm{S}$, Withers $\mathrm{EH}$. Sternal reconstruction with omental and pectoralis flaps: a review of 415 consecutive cases. Ann Plast Surg. 2012 Sep;69(3);296-300. doi: 10.1097/ SAP.0b013e31822af843.

11. Bleichrodt RP, Malyar AW, de Vries Reilingh TS, Buyne O, Bonenkamp JJ, van Goor H. The omentum-polypropylene sandwich technique: an attractive method to repair large abdominal-wall defects in the presence of contamination or infection. Hernia. 2007 Feb;11(1):71-74. doi: 10.1007/s10029006-0174-3.

This article meets the Accreditation Council for Graduate Medical Education and the American Board of Medical Specialties Maintenance of Certification competencies for Patient Care and Medical Knowledge. 